\title{
The Construction of Library Virtual Community
}

\author{
Zhiyin Yang \\ Information and Technology College, Jilin Agricultural University, Jilin, China \\ 23133124@qq.com
}

Keywords: Network; Virtual Community; Library

\begin{abstract}
As a new interactive platform, the network virtual community has incomparable advantage over the traditional interactive platform. Use modern information technology, such as the Web3.0 P2P network technology, SQL server database. Based on this theory, apply virtual community in modern digital library construction. To explore the new way of the construction. Describes in detail the construction of virtual community library. Such as 3D game and task driver way's.Analysis of its problems which during the development process as well as the solution. Finally a successful library virtual network community needs to have what kind of functions and facilities.
\end{abstract}

\section{Introduction}

In recent years, the rapid development of Internet in China, many libraries have access to the network, and on the network to open their own home page and related sites, but a considerable number of libraries have not yet recognized the network virtual community in the library user education function And the role. Virtual community construction in the development of the network has been more mature. Now the network is more popular virtual community website in China are the Tianya, Netease, Mop, West Temple alley, Baidu Post it, Renren and so on. Mainly in foreign countries Facebook, Second Life, Twitter and so on. The success of these virtual communities will provide valuable experience for their applications in the library. The establishment of library virtual community is of great significance, not only to meet the needs of students in school, but also to become the general public to enhance culture, access to knowledge of the important window, so the library virtual community to other existing virtual community form of learning.

\section{Virtual Community Overview}

Since the 1990s, the continuous development of information technology has made the concept of community extended to the virtual network, thus promoting the birth of the virtual community. The earliest definition of a virtual community is made by Rheingole, which defines it as "a group of people who communicate with each other primarily through a computer network that they have a certain degree of awareness and sharing The degree of knowledge and information, to a large extent as a friend to treat each other, and thus formed the group."Virtual community is based on SNS (Social Networking Service) a social network service model. From the point of view of network technology, virtual communities, also known as BBS (Abbreviations for Buletin Board System), forums. From the sociological point of view, refers to the Internet users in the electronic network space for frequent social interaction with the formation of cultural identity of the community and its activities. As the virtual community is conducive to promoting communication between groups of users, in promoting the user distributed learning and research work has a unique advantage [1].

\section{Analysis on the Necessity and Feasibility of Library Virtual Community Construction}

Necessity Analysis. With the development of digital tide, the library's service mode also needs to be adjusted. Web3.0 will use Mashup technology to integrate user-generated content information, which makes the content information more obvious and easy to retrieve. And its network model will achieve different terminal compatibility, from the PC Internet to WAP mobile phones, PDA, set-top 
boxes, dedicated terminals, not only in the Internet on a single terminal. Web3.0 will make the Internet age into a new height, and this will undoubtedly affect the knowledge management industry: the library.

With the emergence of Web3.0, marking the library will also from Lib2.0, leap into Lib3.0 era. The library will no longer be a simple borrower, and will also incorporate new services that include digital services, digital information retrieval, and digital management. And the construction of virtual community is particularly urgent and important.

The traditional library is mainly based on the paper as the carrier of knowledge storage. With the continuous development of information technology, paper media will gradually be replaced by digital information media. Digital information has the characteristics of low storage cost, easy management and easy access. In the traditional library model, the user can only accept the object as information. In the virtual community, each user may become the main body of information creation and dissemination. This will undoubtedly provide users with more convenience in the library virtual community to find resources and access to information [2].

The traditional way of interacting with libraries is simply borrowed and also, the model is single. In the new virtual community, with the help of network information technology, we can do between customers and customers, customers and managers, managers and managers continue to communicate between. Enrich this large "database" information. So that the dissemination of information and inquiries no longer boring, change the more "human touch."

Feasibility analysis. Web 3.0 era of network access will be very fast; Web site will be more open to provide their own API will be the standard site configuration; information association through semantics to achieve, the search ability of information will reach a new height. The technical means of library virtual community construction has become more and more mature. Library can not only follow the example of Netvibes, Facebook, IGoogle, Live, the domestic Sohu Blog also introduced Netvibes and so on, try to do open blog Widget platform, enrich their own network information. And dedicated to the library Lib3.0 knowledge sharing system, but also for the construction of the library virtual community provides a strong guarantee [3].

The library has a large user base, such as college students, employees of various enterprises, various local national libraries. Especially the major colleges and universities, not only focus on a large number of students and teachers, is conducive to the collective wisdom of the play, but also for the construction of the library virtual community to provide strong technical support [4]. Compared with the above more concentrated user base, the library virtual community more users will be relatively dispersed, which also meet the characteristics of network users, and the relationship between the network users will be established in their interaction with the library Contact, thereby indirectly increasing the user base of the library.

Internet library virtual community digital resources, compared with the traditional paper resources of the dominant library has many advantages. The use of digital technology to deal with and store a variety of illustrations and literature library, in essence, is a multimedia production of distributed information systems. It uses a variety of different carriers, different geographical information resources with digital technology storage, in order to facilitate cross-regional, object-oriented network query and dissemination. It involves the whole process of information resource processing, storage, retrieval, transmission and utilization. With a small footprint, easy to retrieve, the use of high characteristics [5].

\section{The Composition of the Library Virtual Community}

Collection of Electronic Resources Query System. In the informationized library virtual community, we will liberate the collection resources from the limitations of the traditional paper carrier, into the electronic version, one to save costs, and secondly to establish a more complete and reliable real-time query system. Traditional query system time and labor, while consuming a lot of resources at the same time, but not the expected results. The network community can use the network database, SQL Server and other tools for quick retrieval, to help users with the least time to obtain the most needed literature. 
Community Interactive Systems. Network era virtual community rich and colorful, such as foreign MSN, Facebook, all the domestic network, QQ and so on. The starting point is to make people break through the space constraints, remote communication, and the construction of the library virtual community can not be separated from a complete social user groups, this group constitutes the basic framework of the virtual community, and as time goes by Continue to grow and develop, to achieve self-growth. It should have the general virtual network community all the features, and according to the different needs of users, real-time from the database to retrieve the user needs information.

Communication Tools. Virtual community relying on Web2.0 and Web3.0 core technology, combined with a variety of communication tools to achieve the Internet content of the intelligent and aggregation, a variety of terminal platform compatible applications[6]. Virtual community main communication tools are as follows:

Instant messaging software (IM). Such as QQ MSN and other software, analog live dialogue, allowing two or more people to use the network in a timely manner to pass text messages, break the space constraints.

Blog (blog). Blog is a rich and colorful personalized display and an integrated platform for users to easily through the network, quickly publish their own experience and so on.

Easy Information Aggregation (RSS). RSS is a technology to synchronize the content of the site, so that users do not have to browse the web page to find information, as long as the need to subscribe to an RSS reader, once the relevant content is updated, RSS will automatically notify the user [7].

Book Mark. Network with a document classification, navigation and other functions. Users can according to their own interests and ideas will be scattered on the network of information resources for sorting and storage, easy access.

Wiki. Wiki is a collaborative publishing environment to provide the site, providing multi-person maintenance, everyone can express their views, with simple and open and other advantages.

Tag (Tag). Tag is a more flexible log sorting method, which can add one or more tags for each $\log$, and then automatically connect to the same Tag of the log, and thus with other users to produce more contact and communication.

\section{The Construction Mode of Library Virtual Community}

3D Page Tour of the Library Virtual Community Construction Model. At present, the library provides the services are the first place in the user, more and more focus on user experience, based on 3D page tour of the library virtual community construction, it is the use of this concept to design.

Web games originated in Germany, it uses php, asp, perl and other interpretation of language and Flash technology, computer hardware and other game environment requirements are relatively low, and no need to download the client or plug-in, the use of these features, Library virtual community construction has become possible [8].

The design and implementation of the game should be user-centric, around the needs of users to start, at the same time to be able to fully and thoroughly express the reality of the library functions, and real library functions to achieve a comprehensive integration. Based on the 3D page tour of the library virtual community to achieve the functional modules are as follows:

Communication is one of the main functions of the library virtual community. The current page tour of the main means of communication through the system comes with IM to achieve online chat. In order to facilitate the user, the chat model to make the appropriate classification, divided into the world, camp, guild. The world is the whole library virtual community, the camp is the same hobby people together to form the organization, and the guild is divided in the camp of a group.

The reference function of the library virtual community based on the 3D page tour can be reflected in many of the details of the page tour. Such as the user to complain and give suggestions, you can consult the system community management staff that the library's reference counselor, the reference to the counselor's questions can be in the same window "My question" column in chronological summary, The formation of the form of $3 \mathrm{D}$ books to store the FAQ, to facilitate the 
reader to use. For the library virtual community some of the conventional issues, can be very detailed in the relevant modules set up common problems help, guides, books, Raiders, etc. for users to read the use.

Based on the 3D page tour of the library virtual community information active push function is very powerful. The community through the page rolling broadcast module to push a series of information to the user, for example, the expired book reminder also in the form of 3D text rolling, the community all online members can be seen, if I am not online, the students can help to tell. New books on the shelves, books recommended, guide, database trial, library news, opening and closing of the notice can be broadcast through the initiative to push. After getting the push information, the reader can $\log$ in to the $3 \mathrm{D}$ page to enter the library to view the details.

Task-driven Library Virtual Community Construction Model. If only the library virtual community as a physical library virtual reproduction, it is difficult to attract the users' continued attention, and the network virtual world will allow users to produce a sense of loneliness. Therefore, in the virtual community design into a certain learning tasks can improve the user's participation.

First of all, the user to complete the registration into the virtual community, according to the tips to familiar with the virtual community environment, but also for the late users skilled use of virtual community resources to prepare. After the user completes the task of familiarity with the environment, there will be accumulated experience, to achieve the default experience value to complete the library resources to borrow. Users can also achieve a certain degree of integration, the establishment of their own learning group or other forms of interest groups, the formation of a certain size in the group, there will be other experience value reward. Thus gaining higher authority.

Second, the task design can be more interesting, to attract the user's participation, such as electronic books to produce audio books, and readers around the world and so on. These interesting and challenging tasks can stimulate learners' interest in learning and gain knowledge in their participation in mission activities.

In addition, the library virtual community and college disciplines combined learning, teachers reserve the task so that students in the library virtual community to complete, you can change the teaching methods, so that the library resources are effectively used.

\section{Problems to be Concerned in the Process of Library Virtual Community Operation}

The construction of the library virtual community is not complete overnight, it can be completed overnight, it still exists many problems, such as the purpose of development is not clear, the lack of adequate network of virtual space moral consciousness and so on. If these problems can not be resolved in a timely manner, the community and the community, facing the risk of collapse, so how to prevent the occurrence of these things at the source has become a library virtual community construction and maintenance process of the most important.

Clear Development Purposes. The establishment of the library virtual community should not be blind, from their own needs, to avoid large and complete, should try to be fine and fine. Should take full account of the actual situation of the library, according to the actual needs of users, widely listen to the views of user groups. In the construction process to pay attention to the virtual community compatibility and scalability, can not blindly expand the space, but also can not rest on its laurels, to focus on sustainable development. The biggest difference between the library virtual community and other virtual communities is that they should be nonprofit. As a public institution, the library has the responsibility and obligation to do their jobs and avoid receiving too much business elements and affecting the library's Normal operation, so that the public welfare has changed the taste.

Code of Moral Awareness. The high degree of freedom of the Internet world makes many network users a network as a public place without any constraints, the lack of moral phenomenon in the network society is particularly serious. And if not to be governed, is bound to affect the network environment, so that the virtual space environment deteriorating. Therefore, the introduction of the relevant network law on the network behavior of the norms and constraints become imperative. In the user management, in addition to managers to strengthen the necessary management efforts, 
update management tools, we should from the source, that is, the user itself to conduct moral management. In practice, we will ask the user to verify the real name. Require users to register first, fill in the real information. Enhance the sense of network responsibility, so that every user really realize that their words and behavior on the network is also subject to legal and moral constraints. Librarians also go deep into the user to, humbly listen to user comments, timely feedback.

Information on the Human Defense of Pollution. At the same time, we should pay attention to the construction of humanistic defense in the virtual community of the library, and construct it in the light of the actual situation. The goal should be conducive to the stability of the community and the healthy development of the community. The library virtual community should give each user more freedom to create a relatively open and equal communication environment. Construction process should pay attention to regulate the user groups in the community words and deeds, is strictly prohibited illegal, the emergence of bad information. At the same time in the management of the construction should be more to develop some incentives, not only help community members to participate actively, and actively offer advice for the construction of the community, but also conducive to the formation of a mutual supervision between members of the atmosphere [9]. The concept of community management should be better for community members, so we should strive to create a green virtual learning environment for members to acquire new information resources. The library virtual community is a place to provide services, its purpose is people-oriented, therefore, human defense should not be virtual members of the community as enemies, but should create conditions to guide users in the moral, legal aspects of a correct understanding, so that they in-depth Experience some of the social norms of ethics and legal [10]. So as to solve the problem of information from the root causes of pollution, is conducive to the healthy development of virtual communities.

\section{Conclusion}

The library virtual community has the characteristics of openness, sociality and the convenience of resource acquisition and the broadness of resource audience. 3D web games can be used as a platform to build a library virtual community that guides students to read and delight their students' physical and mental roles. Or based on task-driven, to establish a task-driven library virtual community that enhances students' interest in reading. Both are based on user needs as the core, which is the library under the new technical conditions of the service function of innovation. It is also the key to the success of the community when the cultural, humanistic defense and virtual community of the library virtual community are put into use after the operation.

\section{References}

[1] C.P. Hu: Modern Library and Information Technology, Vol. 11(2007) No.7, p.87.

[2] L.Q. Sun: Library Science Research, Vol.5 (2005) No.3, p. 5.

[3] S.M. Wang: Library Science Research, Vol.12 (2011) No.11, p.28.

[4] L.J. Peng: Journal of Library Science, Vol.8 (2013) No.7, p.66.

[5] Y Long: Journal of Library and Information Sciences in Agriculture, Vol.11 (2011) No.6, p.36.

[6] S. Cai: Computer Application Research, Vol.6 (2004) No.6, p.258.

[7] J.J. Zhou: Journal of Library and Information Sciences in Agriculture, Vol.7 (2010) No.25, p.71.

[8] T. Xv: Software Guide, Vol.7(2012) No.15, p.79.

[9] L.Y XV: Journal of Liaoning Administration College, Vol.7 (2009) No.9, p.57.

[10][10] L.P. Cristina, P.A.: Information Processing and Management, Vol.12 (2003) No.39, p. 669. 